\title{
Multiobjective based Event based Project Scheduling using Optimized Neural Network based ACO System
}

\author{
Vidya Sagar Ponnam \\ Research Scholar, \\ CSE Department, \\ Sri Krishnadevaraya University, \\ Ananthapuram, India
}

\author{
N.Geethanjali, PhD \\ Associate Professor \& Head Dept. of Computer \\ Science \& Technology , \\ Sri Krishnadevaraya University, \\ Ananthapuram, India
}

\begin{abstract}
In any software project management, developing third party software tools and scheduling tasks are challenging and important. Any software development projects are influenced by a large number of activities, which can greatly change the project plan. These activities may form groups of correlated tasks or event chains. Assessment planning is a crucial challenge in software engineering whose major goal is to schedule the persons to different tasks in such a way that the quality of the software product is optimal and the cost of the project should be minimum. In the traditional approach an event dependent scheduler ant colony optimization is applied on task scheduling. The ACO will develop an optimized plan, in the form of matrix, from all the iterations. And from that plan the EBS(Event Based Scheduler) will develop schedule based on events. ACO solves the problem of project scheduling, but it does not consider the updated task allocation matrix. The ACO is not a satisfactory model to solve the problem of project scheduling. The traditional ACO system also indicates the problem of allocating the identical activity for several numbers of employees in varying periods.In this proposed work, an improved ACO approach to optimal global search using a neural approach was introduced to schedule multiple tasks. An activity with specified number of tasks and relevant resources can be optimally scheduled using multi-objective approach. When an uncertain event occurs the remaining resources will be effectively calculated, also the remaining tasks to complete. And again a new schedule will be generated according to it. An enhanced Entropy method can be used to denote the level about how much threshold or information has been figured out into the pheromone trails and subsequently the heuristic parameter can be improved accordingly.
\end{abstract}

\section{Keywords}

Project management,scheduling,task partition.

\section{INTRODUCTION}

Software project professionals acknowledge the importance of managing uncertainties. The iterative refinement approach, detection and research of possible negative aspects and utilization of other best approaches can scale down uncertainties and help to bring the project as per the scope,standared time estimate and price $[1,7]$.

Yet, software professionals haven't been always expert with controlling approaches and probabilistic scheduling or choose as redundant overhead. Modeling the event schedule with uncertainties in the direction of the planning part continues to be crucial since it allows the supervisor to estimate feasibility of the analyze the project. Due to significance and challenges of project planning, there exists a need for better computer aided utilities for software project planning in recent instances. To plan a software project, the project supervisor must assess the project operations and determine the project plan and supply allocation. To boost more fascinating tools and kinds conventional project planning approaches ought to be moreover extended. In this particular paper, a good attitude for the task scheduling and personnel allocation challenge in project planning utilizing an optimized ant colony optimization (ACO) algorithm is recommended.

Bellenguez and Ne'ron [8], [9] utilized a multiskill scheduling method by extending the normal RCPSP method. The technique considers both the issues of human resource allocation and endeavor scheduling, and takes the ability proficiency of personnel and supply multiple tasks into consideration. Tabu search space (TS) [10], branch and certain [10], and GA [11] are developed for the method. In each of the kinds, there's an prediction that pre-emption is not allowed. As outlined, this assumption reduces the reliability of human source allocation for software duties. Exercise preemption in application tasks is simply regarded in a few research. In Chang's recent proposed system [3], he improved his conventional scheduling attitude by introducing a 3D similarity matrix illustration, specifying the workload activity of each worker for each undertaking on every time period. Although this illustration is far more bendy, it makes the seek area house very huge and suffers from the matter of task of workloads. In this paper, an competent procedure for project scheduling and human source allocation challenge is utilized. It also considers the unsure pursuits handling.

\section{RCPSP- Resource Constrained Project Schedule Challenge}

RCPSP asks a few crucial: Handed a mess of actions, a group of resources, along with a measurement of effectivity, so which is the ideal system to assign the resources onto the actions in an approach that the overall performance is maximized? Which is the most effective technique to assign the resources into the actions at specific instances, so that every a part of the restrictions are satisfied and naturally the ideal goal measures are generated?

RCPSP can simply be thought as follows: particular actions that should be executed a multitude of resources with which to participate in the activities, specific restrictions, which ought 
to be satisfied a bunch of targets with which need to be finished [1-5]

In the paper[6] an optimal PSO approach for resourceconstrained project scheduling issue was implemented. Enhancements depends on the simple particle swarm optimization incorporate: the particle is initiated by heuristicrules to reinforce the high qualitative particles; weight was selfadapted with the iteration of the approach to de-accelerate the velocity of particles; crossover mechanism of filtering approach has been utilized to particle swarm to permit the trade-off excellent traits between each particle. The target of this study was to lessen the overall software project span time. Computational software project circumstances of PSPLIB display that this improved particle swarm optimization approach was valuable as in contrast with other heuristic techniques. In the YanLiu launched a new fuzzy filtering algorithm for software project scheduling to overcome the challenge with supply constraints and overall project period $[6,7]$.

Firstly, fuzzy set was used to symbolize the uncertainty of undertaking period together with the corresponding evaluation system of the fuzzy variety known as the integral worth method was launched. Second, three filtering operators have been used to look for an approximate shortest software project make span. As a result, this gained knowledge of providing another metaheuristic procedure for fixing resource-constraint software project scheduling challenges with a unclear endeavor period.

In the paper[5] Mohammad Amin Rigi, Shahriar Mohammadi K. N. Toosi implemented a brand new method to supply constrained based software project scheduling issue. A hybrid filtering algorithm such as constraint satisfaction, challenge has been used to find source constrained based project scheduling. GA's endeavor was to locate the ideal schedule. Their method uses constraint satisfaction, challenge with the intention to overcome the present inconsistencies in pursuits priority and resource constraints. A full state constraint satisfaction, challenge with minimum conflict heuristic has been used for fixing priority conflicts and a straightforward iterative constraint satisfaction challenge is used to resolve the original source conflicts.

For multi-objective RCPSP, Viana and Pinho de Sousa [9] implemented and reduce the mean weighted lateness of pursuits and scale down the sum of the violation of original source availability. Also, for the multidimensional RCPSP, Dodin \& Eliman [18] implemented the mixing of the elements of time, rate and quality on what they are known as the "Totally Optimized Project System (TOPS)". The TOPS may well be said a follows: "Determine the beginning and conclude time of project actions (such as period), the fabric ordering and stock suggestions, together with the allocation of human resources and gear to those actions as a way to reduce the entire fee of the project or to optimize other selected measures of performance". They argued that their built-in project scheduling challenge could have less total rate than independently considering this goal in a unmarried goal challenge. A essential investigate TOPS exhibits that the authors didn't consider the effect of restricted resources as clearly studied in RCPSP. A consideration of the tri- nature of project goal (time, fee and quality) allows for a multi dimension method to reaching the final goal where resources are restricted[10-14].

\section{PROPOSED APPROACH}

ACO [10] is a method based on the activity of the task scheduling to find a shortest path from a source task to the destination task. This method uses the activity of the real-ants while looking for the food as optimal tasks. It was examined that the ants place a certain quantity of pheromone in its path while visiting from its source place for the food. Consistently while going back, the ants exposed to follow the same direction marked by the pheromone place and again set the pheromone in its path. In this manner the ants follow the shortest-path are predicted to return much earlier and thus enhance the quantity of pheromone guarantee in its path at a quicker rate than the ants focusing on a longer-path. Yet, the pheromone is exposed to evaporation by a certain quantity at a consistent rate over the certain interval and consequently the paths visited by the ants often, are only kept as evidence by the pheromone place, conversely the paths infrequently visited by the ants are dropped due to the lack of pheromone set on that path and consequently the new ants are planned to follow the often used paths only. And hence, most of the ants initiating their journey can learn from the resources and info left by the formerly visitor ants and are directed to follow the shortest-path instructed by the pheromone deposit. In ACO approach, various synthetic ants construct solutions to the optimization problem and replace information on the quality of these solutions over a communication plan .

\section{Initialization:}

Input :J: Jobs

R: Resources

T: Time

Algorithm : ProjectInit(J,R,T)

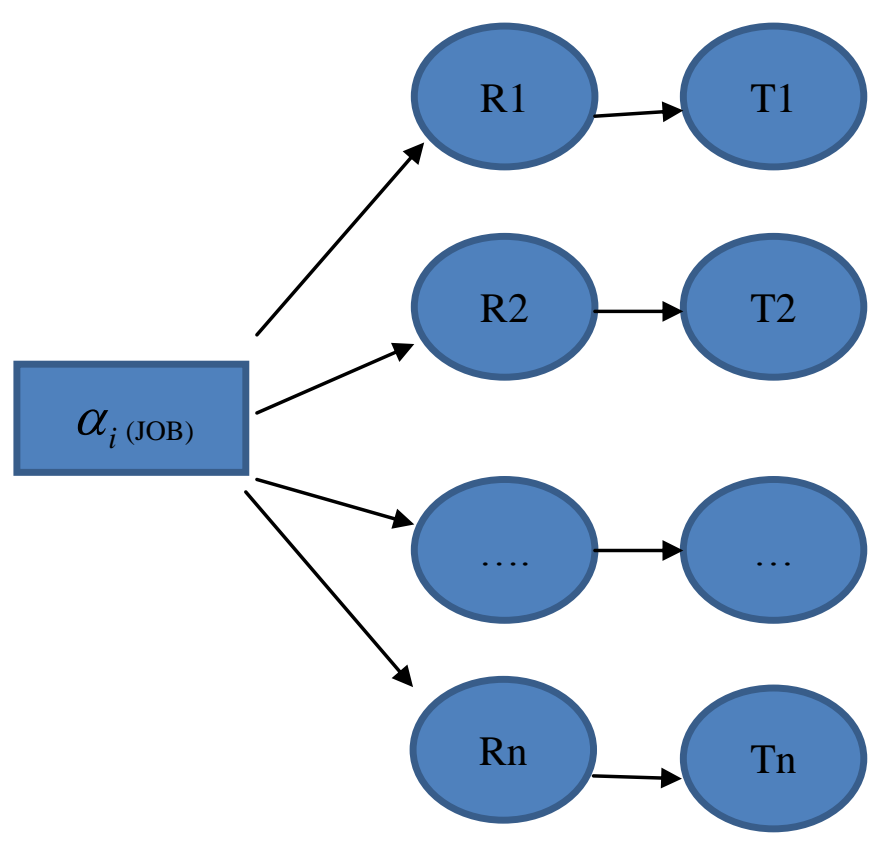

Fig 1. Resource Mapping 
List $\alpha=$ getAll(J);// Get all available jobs

List $\beta=$ getAll(R);//Get all available resources

For each Job in $\alpha$

Do

List $\varphi=$ Check the available resources $(\alpha, \beta)$

Done

Map each job with available resource as Matrix $m_{r t}^{j}$

//check the minimum resource available time

Set the schedule_time $\mathrm{t}=0, \forall i \in \alpha$

For each available resource $\beta$ and available time $\mathrm{T}$

Do

List Temp1:=Min $\left\{\alpha_{i}, \beta, \mathrm{T}\right\} \quad / /$ get all minimum resources

with minimum time.

List Temp2:= $\operatorname{Min}\left\{\alpha_{i}, \beta, \mathrm{T}\right\}$

Multiple jobs resource constrained scheduling mathematically derived as

Optimal scheduling can be achieved using equation 1

minDuration $=\min \{$ Temp1,Temp2 $\}$

s.t $\quad T_{s}^{j} \leq t \leq T_{f}^{j}$

Done

When the improved ACO method begins to operate, the capacity of information on each single path balance with one another, information entropy(ie) is optimal at this time, however an improvement of pheromone on the path, the entropy value will certainly be minimized steadily. In case the entropy is not managed, the entropy will ultimately scale down to 0 , usually, the pheromone on only one path is optimal, and the last solution tends to be erroneous, thus carrying about the incomplete. In order to get over the easily occurred flaws for solving challenging optimization problems with the basic algorithm, a proposed imprioved aco based on optimized squared information entropy is mentioned, applying the heuristic parameter value selection monitored by squared information entropy factor.

1. Basic Initialize Step: Create initial feasible basic solutions and find out the appropriate objective function values;

2. Set primary values of pheromones and other with Temp1 and Temp2.

3. Each ant selects its own path to find the optimal solution. Each ant decides its next node(job task) as per the task selection process and constructs the complete solution matrix.

4. By employing the fitness functionality, each ant evaluates the optimal solution and also estimatet he duration of project, overwork and cost for that project.

5. Classify the all solutions using Neural Network System and Examine the all solutions and decide the most suitable one, revise the pheromone value.

6. Perform repeatedly until the condition is fulfilled. Usually the termination condition is identified by setting the number of iterations.

7. Decide and display the best optimal solution through whichduration and cost is less
Calculate the entropy value of present pheromone trails and then revise the heuristic parameters until the condition is satisfied.

Repeat

Until ant $\mathrm{k}$ has finished

End

In each trail a discrete random variable is selected from the pheromone matrix. The enhanced entropy value of a random variable is calculated as

$$
\operatorname{Ent}(\mathrm{X})=-\sum_{i=0}^{n} p_{i} \log \left(\mathrm{p}_{i}\right)
$$

where $\mathrm{p}_{i}$ denotes the probability of occurrence of each trails in the pheromone matrix. When the probability of the each trail is same then $\mathrm{E}$ will be the optimal and it is defined as

$$
\operatorname{Max} \operatorname{Ent}(\mathrm{X})=-\sum_{i=0}^{n} 1 / \sqrt{r}_{i} \log \left(1 / \sqrt{r}_{i}\right)
$$

We recommend to utilise the squared entropy value as an index to signify the degree about the level information is gained into the pheromone trails and then the heuristic parameter can be updated appropriately.

$$
\begin{gathered}
\operatorname{MaxEnt}(\mathrm{X})=-0.5 * \sum_{i=0}^{n} 1 / \sqrt{r}_{i} \log \left(1 / \mathrm{r}_{i}\right) \\
\operatorname{MaxEnt}(\mathrm{X})=-0.5 * \sum_{i=0}^{n} 1 / \sqrt{r}_{i}\left[\log (1)-\log \left(\mathrm{r}_{i}\right)\right] \\
\operatorname{MaxEnt}(\mathrm{X})=-0.5 * \sum_{i=0}^{n} 1 / \sqrt{r}_{i}\left[0-\log \left(\mathrm{r}_{i}\right)\right] \\
\operatorname{MaxEnt}(\mathrm{X})=0.5 * \sum_{i=0}^{n} 1 / \sqrt{r}_{i}\left[\log \left(\mathrm{r}_{i}\right)\right]
\end{gathered}
$$

\section{EXPERIMENTAL RESULTS}

In this section we present the result of the computations and comparisons with the best conventional approaches. The approach is implemented in java and run on a netbeans IDE. Well known benchmark problem instance sets are used to evaluate the algorithm (PSPLIB, http://www.bwl.unikiel.de/Prod/psplib/index.html). The sets $\mathrm{j} 8, \mathrm{j} 10, \mathrm{j} 30, \mathrm{j} 60$ consist of more than 500 problem instances are used in our experimental study. In our implementation, the neural learning coefficient $\alpha$ is taken as dynamically and the weights are initialized at random process. 


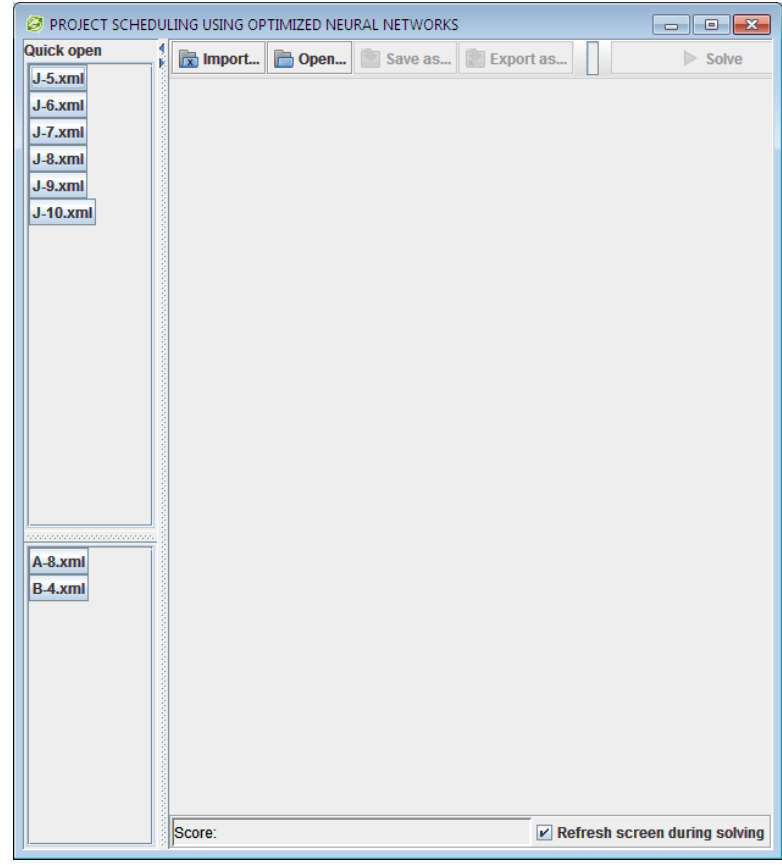

Fig 3. Task related Data Loading

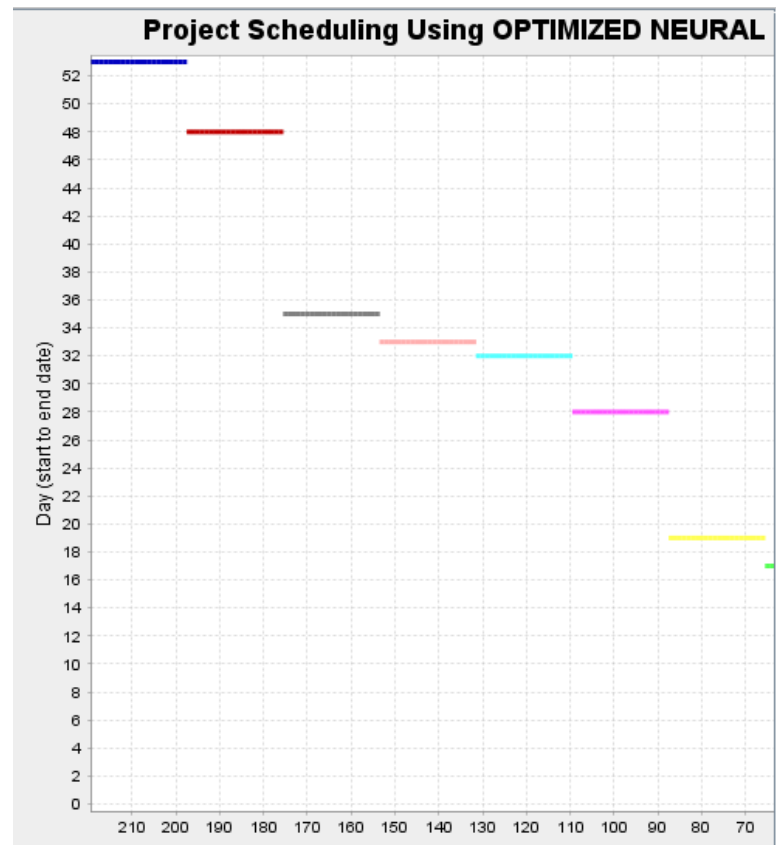

Fig 4. Initial Job Scheduling Before Optimized ACO approach
Project Scheduling Using OPTIMIZED NEURAL NETWOI

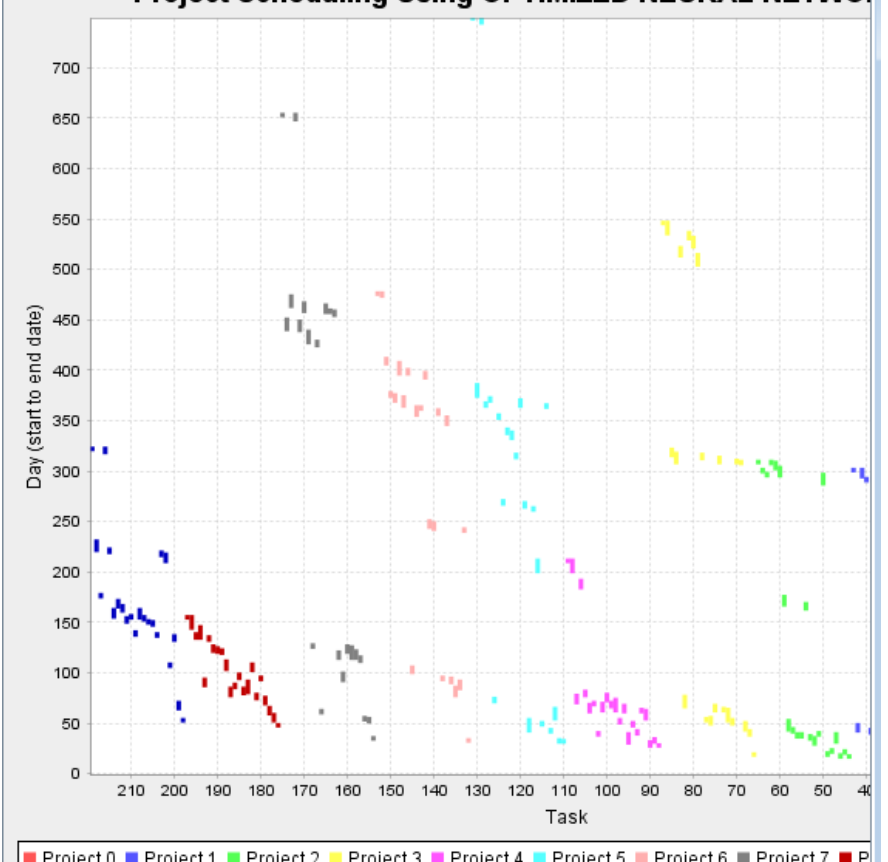

Fig 5. Task Vs Days To complete

\begin{tabular}{|c|c|c|}
\hline & \multicolumn{2}{|c|}{ Performance Analysis } \\
\hline Algorithm & Accuracy & Average Deviations \\
\hline GARCPSP & 0.76 & 0.85 \\
\hline ACORCPSP & 0.82 & 0.89 \\
\hline Proposed & 0.96 & 0.98 \\
\hline
\end{tabular}

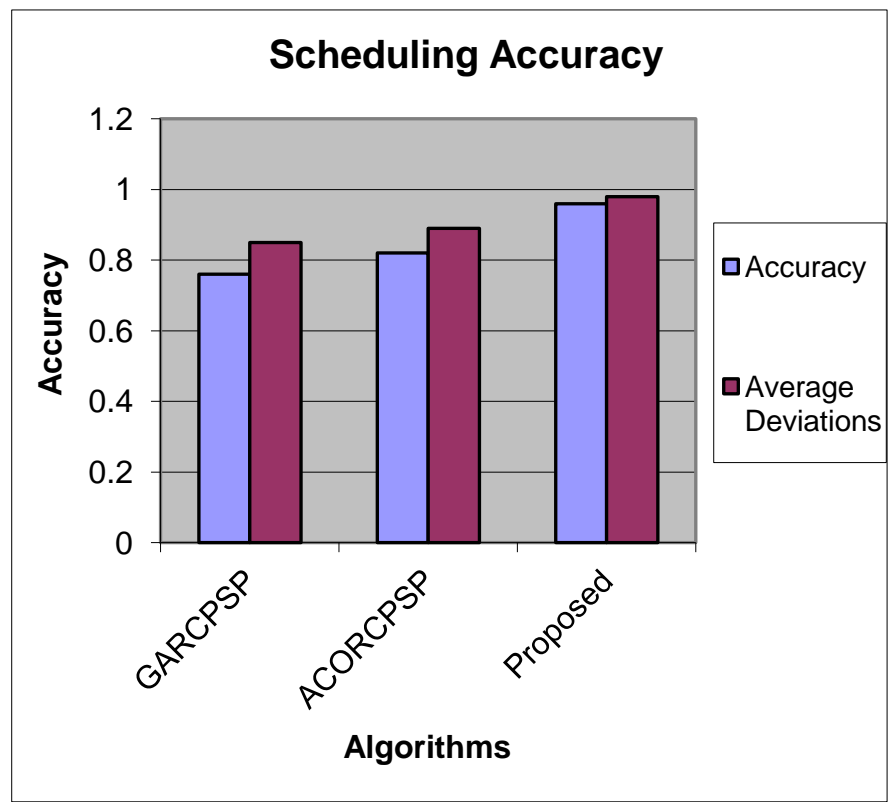




\begin{tabular}{|c|c|c|c|}
\hline & \multicolumn{3}{|c|}{ J60 PROJECT(600 Instances) } \\
\hline Algorithm & Solved & Total & Optimality(\%) \\
\hline GARCPSP & 345 & 600 & 0.575 \\
\hline ACORCPSP & 367 & 600 & 0.611 \\
\hline Proposed & 524 & 600 & 0.87 \\
\hline
\end{tabular}

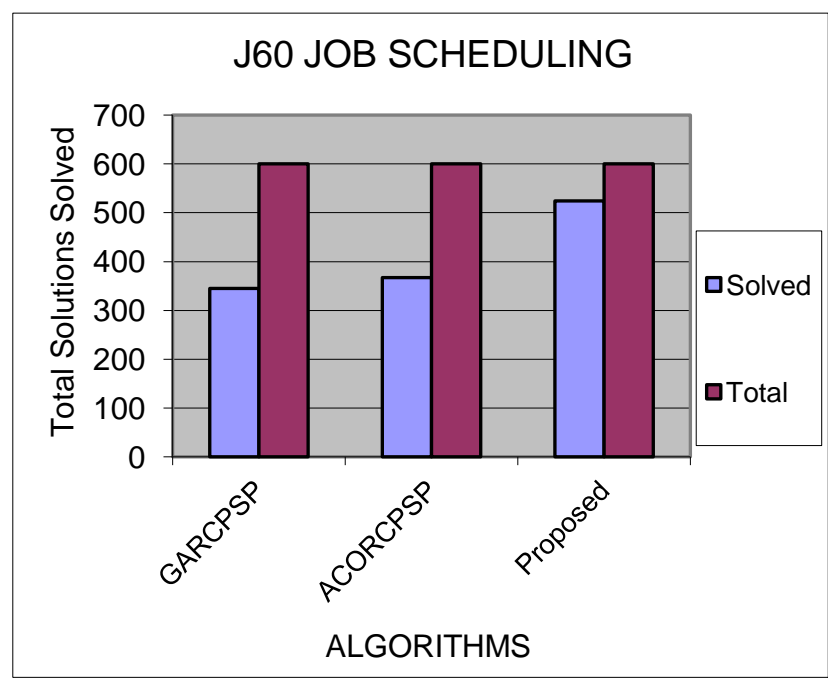

\begin{tabular}{|c|c|c|c|}
\hline & \multicolumn{3}{|c|}{ J8 PROJECT(500 Instances) } \\
\hline Algorithm & Solved & Total & Optimality(\%) \\
\hline GARCPSP & 278 & 500 & 0.575 \\
\hline ACORCPSP & 367 & 500 & 0.734 \\
\hline Proposed & 467 & 500 & 0.93 \\
\hline
\end{tabular}

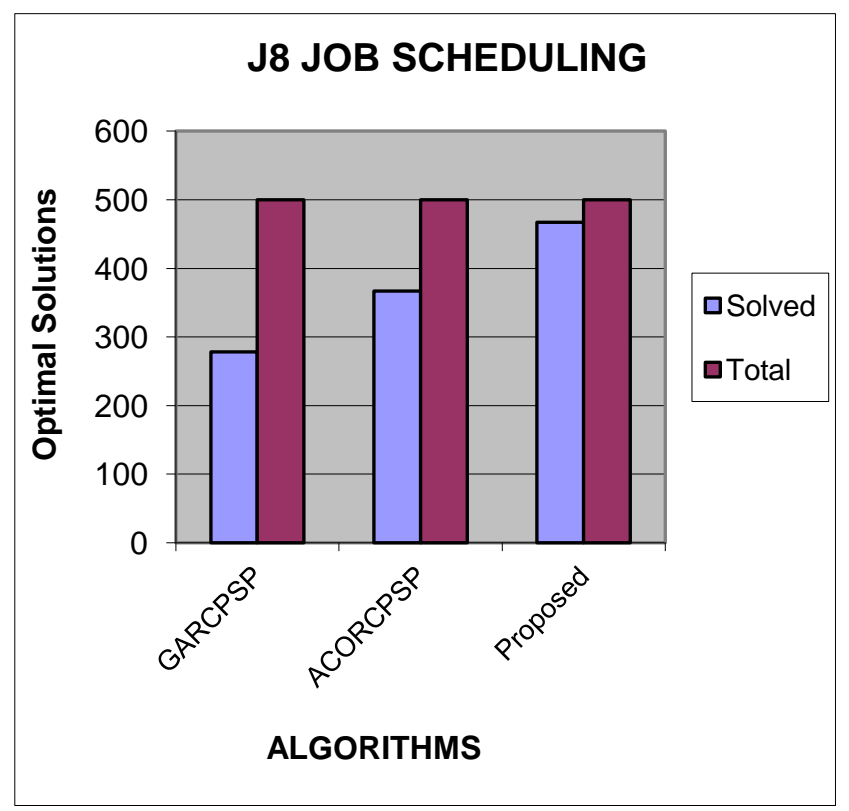

\section{CONCLUSION}

The main objective of this paper is to solve the complicated planning problem, the second one method introduces an event-based scheduler using proposed approach. Both methods have limitation during the project planning and allocation. Experimental results show that the representation scheme with the EBS is effective in small target tasks, and the improved ACO algorithm manages to yield better plans with high statistic-t and mean access time and more stable workload assignments compared with other existing approaches. In this proposed work, an improved ACO approach with optimal global search using neural approach was introduced to schedule multiple tasks. An activity with specified number of tasks and relevant resources can be optimally scheduled using multi-objective approach. When an uncertain event occurs the remaining resource will be effectively calculated, also the remaining tasks to complete. And again a new schedule will be generated according to it. An enhanced Entropy method can be used to denote the level about how much threshold or information has been figured out into the pheromone trails and subsequently the heuristic parameter can be improved accordingly.

\section{REFERENCES}

[1] C. K. Chang, M. J. Christensen, T. Zhang, (2001) "Genetic algorithms for project management", Annals of Software Engineering, Vol. 11, pp107-139.

[2] T. Hanne \& S. Nickel, (2005) “A multiobjective evolutionary algorithm for scheduling and inspection planning in software development project", European Journal of Operational Research, Vol. 167, pp 663-678.

[3] J Leung, ( Ed), Handbook of scheduling: algorithm models and performance, CRC Press LLC; Florida. 2004

[4] E. Alba \& J. F. Chicano, (2007) "Software project management with GAs", Information Science, Vol. 177, pp 2380-2401.

[5] Mohammad Amin Rigi, Shahriar Mohammadi K. N. Toosi Finding a Hybrid Genetic AlgorithmConstraintSatisfaction Problem basedSolution for ResourceConstrained Software project Scheduling University of Technology, Industrial faculty, IT group Tehran, Iran, 2009 International Conference on Emerging Technologies.

[6] Stinson, J.P., Davis, E.W. and Khumawala, B.M., "Multiple Resourceconstrained Scheduling Using Branch-and-Bound", AIIE Transactions, Vol. 10 No. 3, 1978, pp. 252 [10] Xinggang Luo 1,2, Dingwei Wang 2, Jiafu Tang 2, Yiliu Tu 3ResourceConstrained Software project Scheduling Problem, Proceedings of the 6th World Congress on Intelligent Control and Automation, June 21 23, 2006, Dalian, China.

[7] Yan Liu1,2,Sheng-Li zharo2, Xi-Ping Zhang2, GuangQiandu2, A GABased Approach for solving fuzzy siftware project scheduling Proceedings of the Sixth International Conference on Machine Learning and Cybernetics, Hong Kong, 19-22 August 2007.

[8] O. Bellenguez and E. Ne'ron, "Methods for the MultiSkill Project Scheduling Problem," Proc. Ninth Int' Workshop Project Management and Scheduling, 2004. 
[9] P. Brucker, A. Drexl, R. Mohring, K. Neumann, E. Pesch, "Resource-Constrained Project Scheduling: Notation, Classification, Models and Methods," European J. Operational Research, 1999. [10] O. Bellenguez and E. Ne'ron, "A Branch-and-Bound Method for Solving Multi-Skill Project Scheduling Problem," RAIROOperations Research, 2007.

[11] L.Ozdamar, "A Genetic Algorithm Approach to a General Category Project Scheduling Problem," IEEE Trans. Systems, Man, and Cybernetics-Part C: Applications and Rev., Feb 1999.

[12] F. Kazemi, R. Tavakkoli-Moghaddam, "Solving a multi-objective multi-mode Resource-constrained project scheduling problem with particle swarm optimization", International Journal of Academic Research, Vol. 3, pp. 103-110, 2011

[13] F. Ballestin, R. Blanco, "Theoretical and practical fundamentals for multi-objective optimization in RCPSP, Journal of Computers and Operation Research, Vol. 38, No. 1, pp. 51-62, 2011
[14] R. Akbari, V. Zeighami, K. Ziarati, "Artificial bee colony for resource constrained project scheduling problem", International Journal of Industrial Engineering Computations, Vol. 2, pp. 45-60, 2011

\section{AUTHORS PROFILE}

P.Vidya Sagar obtained his M.C.A from Visveswaraiah Technological University,Belgaum.Then he obtained his M.Tech in Computer Science And Engineering from Acharya Nagarjuna University,Guntur and pursuing $\mathrm{PhD}$ in Computer Science and Technology from Sri Krishnadevaraya University,Anantapuram. He is a Professional Member of ISCA. His specializations include software engineering and software reliability, web services and networking.

Dr.N.Geethanjali received her $\mathrm{PhD}$ Degree from Sri Krishanadevaraya University. Andhra Pradesh, India she is working as Head, Department of Computer Science \& Technology, Sri Krishanadevaraya University. Andhra Pradesh, India. She is a Professional Member ACM. Her research interest includes Computer Networks, Cloud Computing, Software Engineering, Programming languages and Data Mining. 